\title{
Optimal insurance design of ambiguous risks
}

\author{
Christian Gollier ${ }^{1}$ \\ Toulouse School of Economics (LERNA, University of Toulouse)
}

January 21, 2013

\begin{abstract}
We examine the characteristics of the optimal insurance contract under linear transaction cost and an ambiguous distribution of losses. Under the standard expected utility model, we know from Arrow (1965) that it contains a straight deductible. In this paper, we assume that the policyholder is ambiguity-averse in the sense of Klibanoff, Marinacci and Mukerji (2005). The optimal contract depends upon the structure of the ambiguity. For example, if the set of possible priors can be ranked according to the monotone likelihood ratio order, the optimal contract contains a disappearing deductible. We also show that the policyholder's ambiguity aversion can reduce the optimal insurance coverage.
\end{abstract}

Keywords: Deductible, risk-sharing, ambiguity, monotone likelihood ratio order.

JEL Codes: D81, G22

\footnotetext{
${ }^{1}$ The research leading to these results has received funding from the Chair "Risk Markets and Value Creation" at TSE, and from the European Research Council under the European Community's Seventh Framework Programme (FP7/2007-2013) Grant Agreement no. 230589.
} 


\section{Introduction}

The ability to share risk among different agents facing imperfectly correlated risks is a crucial element of the functioning of our economies. The recent financial crisis demonstrates the catastrophic consequences of the inefficiencies that plague risk-sharing markets. In this context, insurance markets play an important role to mutualize individual risks. In the absence of transaction costs, full insurance would be optimal in order to wash out these risks through their pooling in financial markets. However, because of asymmetric information, the monitoring of these individual risk transfers usually entails high transaction costs. In some insurance lines, these costs can go as high as $30 \%$ to $50 \%$ of the actuarial value of the policy. When insurance entails such large deadweight losses, it is intuitive that partial insurance is optimal. Various forms of partial coverage can be considered. Insurance clauses such as a proportional retention rate, a straight deductible, or an upper limit of coverage are a few examples.

Arrow (1963, 1965, 1971, 1974) has been the first to examine the problem of the optimal design of the insurance contract. He showed that it has a very specific form when transaction costs are proportional to the indemnity. Namely, the optimal contract is a contract with a straight deductible. For losses below the deductible, the insurer pays no indemnity. For losses above the deductible, the indemnity equals the loss minus the deductible. The intuition of this result is simple: The deductible insurance contract is the best compromise between the willingness to reduce risk and the need to limit the insurance deadweight cost. Any increment of indemnification opportunity should be used to cover the largest uncovered loss. This is because, under risk aversion, the marginal utility of wealth is decreasing. Gollier and Schlesinger (1996) have shown that any contract other than with a straight deductible is dominated in the sense of Second-order Stochastic Dominance (SSD) by the contract with a straight deductible with the same premium. It implies that risk-averse agents should always prefer such a contract. It also implies that the Arrow's result holds in all decision theoretic frameworks in which the preference functional exhibits the SSD property. This was shown by Zilcha and Chew (1990), Karni (1992), and Machina (1995).

The Arrow's result has also been extended in other directions. Raviv (1979) and Blazenko (1985) showed that the optimal insurance contains a coinsurance rule above the deductible when the insurer is risk-averse, i.e., when the individual risk cannot be washed out through 
mutualisation. Raviv (1979) and Huberman, Mayers and Smith (1983) explored the case of nonlinear transaction costs. A convex relationship between the indemnity and the cost can also explain why coinsurance may be optimal above the deductible.

Comparative statics analyses have also been performed in the literature. Mossin (1968) showed that the optimal deductible is positive and decreasing with the degree of risk aversion of the policyholder. Eeckhoudt, Gollier and Schlesinger (1991) examined the impact of a change in the distribution of losses observed by the two parties on the optimal deductible.

Following Carlier, Dana and Shahidi (2003), we extend these analyses by characterizing the optimal insurance contract when the distribution of losses is ambiguous and the policyholder is ambiguity-averse. We specifically consider the case of smooth ambiguity aversion in the sense of Klibanoff, Marinacci and Mukerji (KMM, 2005). There is little doubt that most people face some uncertainty about the distribution of their potential future losses. The estimation of individual probabilities associated to various health hazards often differ from observed frequencies, as documented for example by Slovic (2000) and Liu, Tsou and Hammitt (2007). Similar ambiguous probabilities can be found in other insurance contexts, in particular for low probability events.

We assume that the policyholder is ambiguity-averse, as is documented by Ellsberg (1961) and by many subsequent experimental findings. Several models of ambiguity aversion exist in the literature. Three models have attracted much attention: the maxmin EU model of Gilboa and Schmeidler (1989), the $\alpha$-maxmin EU model of Ghirardato, Maccheroni, and Marinacci (2004), and the smooth ambiguity aversion model of KMM. In this paper, we use the KMM model for its ability to define the notion of ambiguity neutrality, and for its simple way to perform the comparative statics of a change in ambiguity aversion.

Following Klibanoff, Marinacci and Mukerji (2009), Gollier (2011) and Ju and Miao (2012), we show that the effect of the policyholder's ambiguity aversion on the optimal contract is observationally equivalent to a change in her beliefs. This change is endogenous to the choice of insurance contract. The ambiguity aversion puts more weight to the priors which yield a smaller conditional expected utility. This form of pessimism may have various effects on the optimal insurance coverage. The intuition suggests that it should increase the demand for insurance, but we show that this is not true in general. In particular, the demand for insurance will be reduced by ambiguity aversion if the ambiguity is concentrated on the probability of small losses. 


\section{The model and its basic properties}

The policyholder faces a risk of loss $x$. The distribution of the loss is ambiguous. This ambiguity takes the form of a non-unique prior. Following KMM, the distribution function $F$ is parameterized by $\theta$ that can take $n$ possible values $\theta=1, \ldots, n$ with probabilities $\left(q_{1}, \ldots, q_{n}\right) \geq 0, \sum_{\theta=1}^{n} q_{\theta}=1 . F(x, \theta)$ denotes the probability that the loss be smaller than $x$ conditional to $\theta$. These cumulative distribution functions can be discrete or continuous. Their supports are in $\mathbb{R}_{+}$. We suppose that there are at least three possible loss levels with a positive probability in at least one $\theta$.

An insurance contract is a pair $(I(), P$.$) , where P$ is the insurance premium and $I($.$) is the$ insurance schedule, with $I(x)$ denoting the indemnity that is paid by the insurer if the observable loss is $x$. We assume that the indemnity can never be negative: $I(x) \geq 0$ for all $x$. Following KMM, the ex ante welfare of the policyholder selecting this contract is measured as follows:

$$
W(I(.), P)=\sum_{\theta=1}^{n} q_{\theta} \phi\left(\int u\left(w_{0}-x+I(x)-P\right) d F(x, \theta)\right)
$$

where $w_{0}$ is initial wealth. We assume that functions $u$ and $\phi$ are both increasing and concave. The concavity of $u$ and $\phi$ expresses respectively risk aversion and ambiguity aversion. When $\phi$ is linear, this model is equivalent to subjective EU. In that case, the policyholder's expected utility is measured by computing the expectation under $\operatorname{cdf} G$, with

$$
d G(x)=\sum_{\theta=1}^{n} q_{\theta} d F(x, \theta)
$$

We assume that the competitive insurer is ambiguity-neutral and faces linear transaction costs: For each dollar of indemnity, the insurer incurs a cost $1+\tau$. This implies that the indemnity schedule $I($.$) is associated to an insurance premium P$ such that

$$
P=(1+\tau) \int I(x) d G(x)
$$


The integral is the actuarial value of the policy. When $\tau=0$, the insurance premium is actuarially fair. When $\tau$ is positive, it is actuarially unfair. Most of the results presented in this paper can easily be extended to the case where the insurance premium is an increasing function of the actuarial value of the policy.

The problem of the policyholder is to select a feasible contract $(I, P)$ that maximizes her ex ante welfare under the tariff constraint:

$$
\max _{I(.) \geq 0, P} \sum_{\theta=1}^{n} q_{\theta} \phi\left(\int u(w(x)) d F(x, \theta)\right) \quad \text { s.t. } \quad P=(1+\tau) \int I(x) d G(x)
$$

where $w(x)=w_{0}-x+I(x)-P$ is the policyholder's final wealth. Notice that the integrands in the objective function and in the constraint are concave in $(I, P)$. Using standard calculus of variations, the following set of first-order conditions are therefore necessary and sufficient for optimality:

$$
u^{\prime}(w(x)) \sum_{\theta=1}^{n} q_{\theta} \phi^{\prime}\left(U_{\theta}\right) d F(x, \theta) \leq \lambda(1+\tau) d G(x)
$$

for all $x$, with an equality when $I(x)>0$, and

$$
\sum_{\theta=1}^{n} q_{\theta} \phi^{\prime}\left(U_{\theta}\right) \int u^{\prime}(w(x)) d F(x, \theta)=\lambda .
$$

Parameter $\lambda$ is the Lagrange multiplier associated to the tariff constraint. In these two conditions, $U_{\theta}$ denotes the expected utility conditional to $\theta$ :

$$
U_{\theta}=\int u(w(x) d F(x, \theta)
$$

It is useful to reinterpret the two first-order conditions by using the distorted cdf $H$ which is defined as

$$
d H(x)=\sum_{\theta=1}^{n} \hat{q}_{\theta} d F(x, \theta)
$$

with

$$
\hat{q}_{\theta}=\frac{q_{\theta} \phi^{\prime}\left(U_{\theta}\right)}{\sum_{t=1}^{n} q_{t} \phi^{\prime}\left(U_{t}\right)},
$$


for $\theta=1, \ldots, n$. Observe that $\left(\hat{q}_{1}, \ldots, \hat{q}_{n}\right)$ can be interpreted as a distorted probability distribution over the set of possible scenarii. Because $\phi$ is concave, this distorted distribution is biased toward scenario that yield smaller condition expected utility. The cdf $H$ is a distribution function on losses that is biased in favour of losses that are more likely to occur under these adverse scenarii. It is noteworthy that these distortions are endogenous because they depend upon the characteristics of the insurance contract that is purchased by the agent. With these definitions, we can rewrite conditions (5) and (6) respectively as

$$
u^{\prime}(w(x)) \leq \mu(1+\tau) \frac{d G(x)}{d H(x)},
$$

with equality when $I(x)>0$, and

$$
\int u^{\prime}(w(x)) d H(x)=\mu,
$$

where $\mu=\lambda / \sum_{\theta=1}^{n} q_{\theta} \phi^{\prime}\left(U_{\theta}\right)$. Observe that these equations can be interpreted as the firstorder conditions of the optimal insurance problem of an ambiguity-neutral policyholder whose beliefs $H$ differ from the insurer's beliefs $G$. But again, the difficulty of this analysis is that the policyholder's beliefs are endogenous to the selected insurance contract.

The seminal work of Arrow (1965, 1971) describes the optimal insurance schedule in the special case of ambiguity neutrality. It is summarized in the following Proposition.

Proposition 1: Suppose that the agent is ambiguity-neutral, i.e., $\phi$ is linear. In that case, the optimal insurance contract has a straight deductible: There exists $D^{*} \in \mathbb{R}$ such that $I(x)=\max \left(0, x-D^{*}\right)$ for all $x$.

Proof: Under ambiguity-neutrality, $H=G$, which implies that the condition (10) can be rewritten as $u^{\prime}(w(x)) \leq \mu(1+\tau)$ for all $x$, with an equality when $I(x)>0$. Let us define $D^{*}$ in such a way that $u^{\prime}\left(w_{0}-D^{*}-P\right)=\mu(1+\tau)$. Obviously, because of risk aversion, condition (10) is satisfied with $I(x)=\max \left(0, x-D^{*}\right)$ for all $x$.

For any given premium, the indemnity schedule that maximizes expected utility is the one that indemnifies the largest losses in priority. The deductible clause does exactly that. Mossin (1968) showed in turn that the optimal deductible vanishes when the insurance tariff is fair, 
and that it is strictly positive when the premium is unfair. The generalization of this result in the case of ambiguity aversion can be summarized as follows.

Proposition 2: When $\tau=0$, the optimal contract entails full insurance, i.e., $I(x)=x$ for all $x$. When $\tau>0$, there exists a subset of losses of positive measure $G$ such that $I(x)=0$.

Proof: When $\tau=0$, it is easy to check that the first-order condition (10) and (11) are satisfied. Because of full insurance, expected utility does not depend upon $\theta$, so that $H \equiv G$. When $\tau$ is positive, suppose by contradiction that $I(x)$ is positive almost surely (using probability measure $G$ ). This implies that equation (10) is satisfied as an equality, or equivalently, that

$$
u^{\prime}(w(x)) d H(x)=\mu(1+\tau) d G(x)
$$

almost surely. Integrating with respect to $x$ yields

$$
\int u^{\prime}(w(x)) d H(x)=\mu(1+\tau) .
$$

Because $\tau>0$, this is in contradiction with condition (11). Thus, there must exist a subset of losses of positive measure such that $I(x)=0$.

When the insurance tariff is actuarially fair, full insurance is optimal. Ambiguity aversion reinforces risk aversion to generate this intuitive result. When the insurance tariff is unfair, no indemnity is paid in some states. The intuition of this result is simple: If a positive indemnity would be paid in all states, a small uniform reduction in indemnity would increase wealth in all states, because the reduction in premium will be larger than the reduction in the indemnity. Under ambiguity neutrality, the classical result of Arrow tells us that these states with no indemnity are for low losses. This is not necessarily the case when the agent is ambiguityaverse.

In the remainder of this paper, we assume that the insurance tariff is unfair $(\tau>0)$.

\section{The optimal insurance design}

In this section, we will mostly be interested by the characterization of the shape of function I(.). Therefore, we will take $\lambda$ (and $\mu$ ) as given, and we will ignore the first-order condition (6) (or (11)) associated to the premium. An important message of this section is that the 
optimal insurance design strongly depends upon the structure of the ambiguity affecting the loss distribution.

\subsection{The ambiguity is concentrated on losses above $D^{*}$}

Let $D^{*}$ denote the optimal deductible under ambiguity neutrality. We start with a very simple benchmark case, which is described in the following proposition.

Proposition 3: Suppose that the ambiguity is concentrated on losses above $D^{*}$, i.e., $d F(x, \theta)=d G(x)$ for all $x \leq D^{*}$ and all $\theta=1, \ldots, n$. Then, the optimal insurance contract is not affected by ambiguity aversion.

Proof: Consider the optimal contract under ambiguity neutrality. By Proposition 1, it contains a straight deductible $D^{*}$. Because the ambiguity is concentrated on $x>D^{*}$, observe that

$$
\begin{aligned}
U_{\theta} & =\left(1-F\left(D^{*}, \theta\right)\right) u^{\prime}\left(w_{0}-D^{*}-P\right)+\int_{x \leq D^{*}} u\left(w_{0}-x-P\right) d F(x, \theta) \\
& =\left(1-G\left(D^{*}\right)\right) u^{\prime}\left(w_{0}-D^{*}-P\right)+\int_{x \leq D^{*}} u\left(w_{0}-x-P\right) d G(x) .
\end{aligned}
$$

This is independent of $\theta$. It implies that $H \equiv G$. Using the same method than in the proof of Proposition 1, it implies that the optimal insurance contract has a straight deductible, and that this deductible is not affected by ambiguity aversion.

This result shows that the agent is myopic to ambiguous probabilities associated to losses above the deductible. Because these losses are fully covered at the margin, their ambiguous probabilities do not affect welfare. Therefore, they do not affect the optimal contract. This is reminiscent of a result in Eeckhoudt, Gollier and Schlesinger (1991) which states that a mean-preserving spread in the distribution of loss above the deductible does not affect the optimal deductible.

This result also shows that the ambiguity must affect losses below the deductible that is optimal under ambiguity neutrality to modify the optimal design of the contract. If the ambiguity affects small losses, the expected utility conditional to scenario $\theta$ will vary across $\theta=1, \ldots, n$. This will affect the distorted distribution $H$ over the entire support of losses. That will globally modify the shape of the optimal insurance design. 


\subsection{The ambiguity is concentrated on losses below $D^{*}$}

Consider a situation in which the insurance contract does not fully eliminated the ambiguity faced by the policyholder, i.e., the $U_{\theta}$ vary across $\theta$. In this section, we first examine the effect of ambiguity aversion in the indemnity paid in two specific states that exhibit "constant ambiguity". The degree of ambiguity in states $x_{1}$ and $x_{2}$ is said to be constant if the probability to incur loss $x_{1}$ conditional to the loss being $x_{1}$ or $x_{2}$ is unambiguous. Technically, this means that the ratio $d F\left(x_{2}, \theta\right) / d F\left(x_{1}, \theta\right)$ is independent of $\theta$. A special case of constant ambiguity is no ambiguity at all, when both $d F\left(x_{1}, \theta\right)$ and $d F\left(x_{2}, \theta\right)$ are independent of $\theta$. In Proposition 4, we show that the deductible applied to these two potential losses must be the same.

Proposition 4: Consider two losses being $x_{1}$ and $x_{2}$ exhibiting constant ambiguity, i.e., $d F\left(x_{2}, \theta\right) / d F\left(x_{1}, \theta\right)$ is independent of $\theta$. Then, the same deductible is applied to these two potential losses in the optimal contract.

Proof: Because ambiguity is constant over $\left\{x_{1}, x_{2}\right\}$, we have that

$$
\begin{aligned}
\frac{d G\left(x_{2}\right)}{d H\left(x_{2}\right)} & =\frac{\left(\sum_{\theta=1}^{n} q_{\theta} \phi^{\prime}\left(U_{\theta}\right)\right)\left(\sum_{\theta=1}^{n} q_{\theta} d F\left(x_{2}, \theta\right)\right)}{\sum_{\theta=1}^{n} q_{\theta} \phi^{\prime}\left(U_{\theta}\right) d F\left(x_{2}, \theta\right)} \\
& =\frac{\left(\sum_{\theta=1}^{n} q_{\theta} \phi^{\prime}\left(U_{\theta}\right)\right)\left(\sum_{\theta=1}^{n} q_{\theta} d F\left(x_{1}, \theta\right)\right)}{\sum_{\theta=1}^{n} q_{\theta} \phi^{\prime}\left(U_{\theta}\right) d F\left(x_{1}, \theta\right)}=\frac{d G\left(x_{1}\right)}{d H\left(x_{1}\right)} .
\end{aligned}
$$

Let us define $D^{\circ}$ so that

$$
u^{\prime}\left(w_{0}-D^{\circ}-P\right)=\mu(1+\tau) \frac{d G\left(x_{i}\right)}{d H\left(x_{i}\right)} .
$$

Applying condition (10) implies that $I\left(x_{i}\right)=\max \left(0, x_{i}-D^{\circ}\right)$ for $i=1,2$. This means that the same deductible $D^{\circ}$ is applied to the two losses $x_{1}$ and $x_{2}$.

By transitivity, if several losses exhibit constant ambiguity, the same deductible will be applied to all. An extreme version of Proposition 4 has been presented by Alary, Gollier and Treich (2011). They assume that the probability distribution of losses is unambiguous conditional to $x>0$. The only source of ambiguity is about the probability of loss. They show that the optimal insurance contract has a straight deductible. This can be seen as an 
application of the above proposition, since all states with $x>0$ exhibits constant ambiguity in their model.

In the following proposition, we present another simple application of Proposition 4. Following KMM, the degree of ambiguity aversion is locally measured by $-\phi^{\prime \prime}(u) / \phi^{\prime}(u)$. We say that the degree of ambiguity aversion is small if there exists a constant $A$ close to zero such that $-\phi^{\prime \prime}(u) / \phi^{\prime}(u)$ is smaller than $A$ for all $u$.

Proposition 5: Suppose that the ambiguity is concentrated on losses below $D^{*}$, i.e., $d F(x, \theta)=d G(x)$ for all $x \geq D^{*}$ and all $\theta=1, \ldots, n$. Then, if the degree of ambiguity aversion is small enough, the optimal insurance contract contains a straight deductible: There exists a scalar $D^{a} \in \mathbb{R}$ such that $I(x)=\max \left(0, x-D^{a}\right)$ for all $x$.

Proof: See the Appendix.

Observe that this result shows that the optimal design of the insurance contract is preserved, but only if ambiguity aversion is not too large. For larger degrees of ambiguity aversion, the presence of ambiguity on the probability of small losses may imply that the policyholder will want to get some positive indemnity for some of them. Observe also that, contrary to the case where ambiguity is concentrated to losses above $D^{*}$, the optimal deductible will in general be affected by the presence of ambiguity below the deductible. The direction of this impact is examined in the second part of this paper.

\subsection{Two-state ambiguity}

We have learned from the previous two subsections that the shape of the optimal contract will be affected by ambiguity aversion only if two conditions are satisfied. First, some losses below the deductible must have an ambiguous probability. Second, some losses above the deductible must have a heterogeneous degree of ambiguity. In this subsection, we examine the simplest ambiguity structure that combines these two conditions. Consider two losses $x_{1}$ and $x_{2}>x_{1}$ having respectively probability mass $p_{1 \theta}$ and $p_{2 \theta}$ conditional to $\theta$. We assume that ambiguity is concentrated on these two states, i.e., that $d F(x, \theta)=d G(x)$ for all $x \notin\left\{x_{1}, x_{2}\right\}$ and all $\theta=1, \ldots, n$. Because $F(., \theta)$ is a cdf, we must have that, for all $\theta$, 


$$
p_{1 \theta}+p_{2 \theta}+\int_{x \notin\left\{x_{1}, x_{2}\right\}} d G(x)=1 .
$$

This condition shows that with a two-state ambiguity structure, the degree of ambiguity cannot be constant across the two ambiguous states. To make the problem interesting, we examine the case in which the smaller ambiguous loss is below $D^{*}$.

Proposition 6: Consider the ambiguity structure with two ambiguous losses, $x_{1}$ and $x_{2}$ such that $x_{1}<D^{*}$. The optimal insurance contract has the following properties:

i. The same straight deductible $D_{0}$ is applied to all unambiguous losses: $I(x)=\max \left(0, x-D_{0}\right)$ for all $x \notin\left\{x_{1}, x_{2}\right\}$;

ii. $\quad$ No indemnity is paid in the smaller ambiguous loss $x_{1}: I\left(x_{1}\right)=0$;

iii. A deductible $D_{2}$ is applied to the larger ambiguous loss $x_{2}$ such that $x_{1} \leq D_{2} \leq D_{0}$.

Proof: See the Appendix.

The main message of this proposition is that when only two losses are ambiguous, this tends to reduce the optimal indemnity for the lower ambiguous loss, and to raise the optimal indemnity for the larger ambiguous loss. For this larger loss, ambiguity aversion reinforces risk aversion to raise the demand for insurance coverage. It is noteworthy that this increase in the indemnity of the larger ambiguous loss does not reverse the ranking of final wealth, in the sense that it is larger conditional to loss $x_{1}$ than conditional to $x_{2}>x_{1}$.

The existence of an ambiguous loss that is not indemnified implies that the policyholder bears some ambiguity about the distribution of final wealth. Because she is ambiguity-averse, the policyholder is willing to take actions that reduce this ambiguity. A marginal increase in the indemnity of the larger ambiguous loss is useful because its positive impact is the largest on the expected utility of the most adverse scenarii $\theta$, which are those with the largest probability for this large loss. Because the probability of the larger ambiguous loss covaries positively with the conditional expected utility, raising the indemnity for this larger ambiguous loss reduces the uncertainty.

Another way to interpret this result is to rely on distorted beliefs. Ambiguity aversion raises the implicit probability of the $\theta$ associated to the smaller conditional $U_{\theta}$. Because the net wealth is 
smaller conditional to the larger ambiguous loss, these $\theta$ are those associated to the larger conditional probabilities $P\left[x=x_{2} \mid \theta\right]$. To sum up, ambiguity aversion distorts beliefs in favour of scenarii corresponding to larger probabilities associated to $x_{2}$. This implies that the distorted cdf used by the policyholder puts more probability weight on loss $x_{2}$ than the ambiguity-neutral insurer. This form of pessimism on the side of the policyholder raises the optimal indemnity of that specific loss.

To illustrate, consider the following numerical example. The policyholder with initial wealth $w_{0}=11$ faces a risk of loss $x \in\{0,1, \ldots, 10\}$. She is risk-averse with utility function $u(c)=\sqrt{c} / 2$. There are two plausible cdf for the loss. The more favourable cdf has a no-loss probability of $1 / 2$. It allocates a 0.05 probability to all other states. The less favourable cdf puts a probability $1 / 2$ to loss $x=8$ and a probability 0.05 to all other states. The two cdf are equally likely: $q_{1}=q_{2}=1$. The loading factor $\tau$ equals $11 \%$. We solved the problem numerically using Mathematica. Under ambiguity neutrality, the optimal deductible is $D^{*}=4.14$, and the optimal contract is depicted by the bullet points in Figure 1. Thus, this example illustrates the two-state ambiguity structure, with one ambiguous loss below the deductible, and the other ambiguous loss above the deductible. In Figure 1, the plain curve describes the optimal contract when the policyholder is ambiguity-averse with function $\phi(u)=-e^{-u}$. We see that the ambiguous loss $x=8$ is better covered than under the application of deductible $D_{0}=3.03$ that applies for all other loss levels. In fact, a deductible $D_{2}=3.77$ applies for that specific loss. 


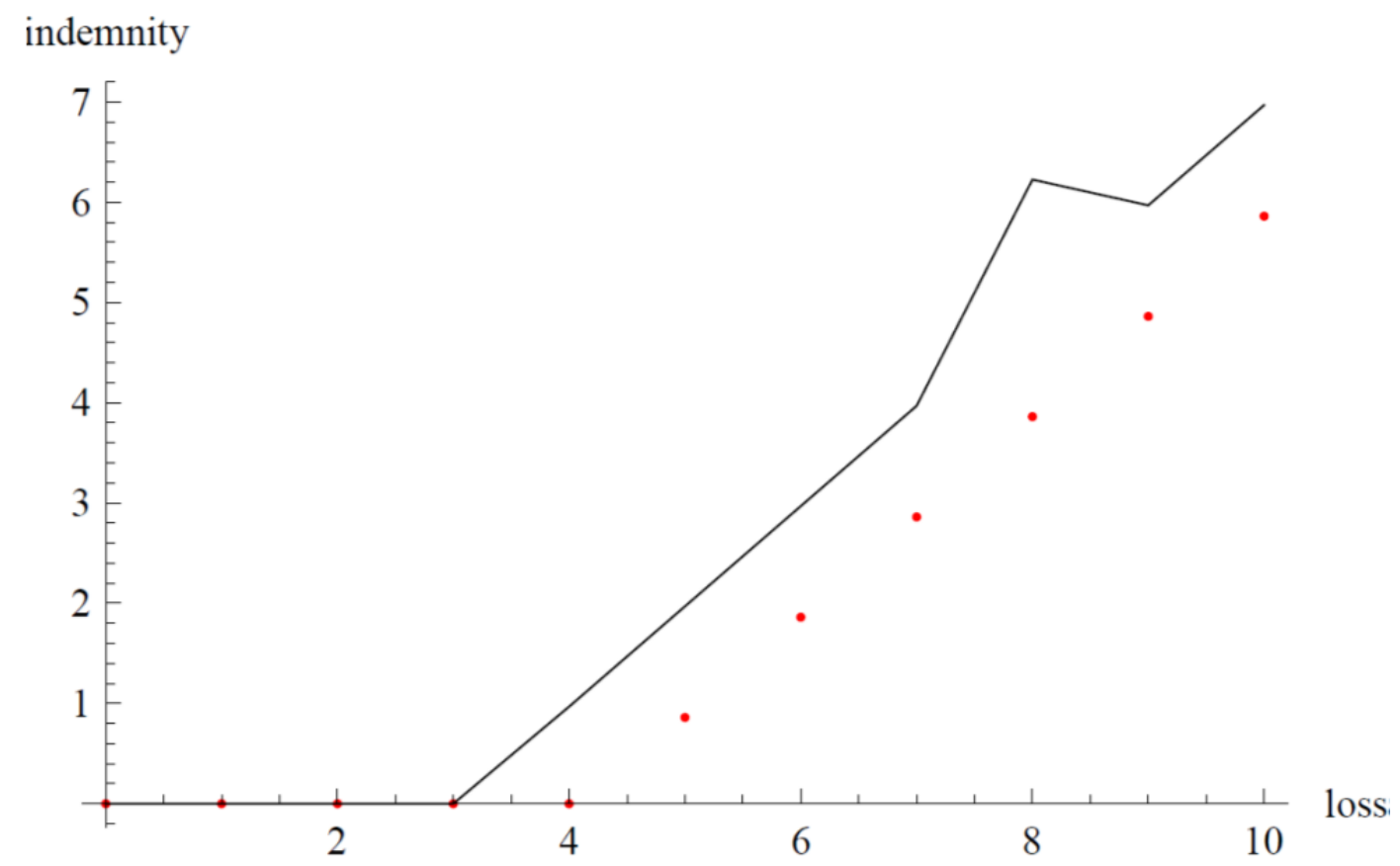

Figure 1: Optimal contract under a two-state ambiguity structure. The support of loss is $\{0,1, \ldots, 10\}$. There are two equally likely cdfs, respectively with probabilities $(1 / 2,0.05, \ldots, 0.05)$ and $(0.05, \ldots, 0.05,1 / 2,0.05,0.05)$. We also assume $u(c)=\sqrt{c} / 2, w_{0}=11$ and $\tau=0.11$. The dots correspond to the optimal insurance schedule under ambiguity neutrality, whereas the plain curve is the optimal contract if $\phi(u)=-e^{-u}$.

\subsection{One-against-all ambiguity}

Another interesting ambiguity structure is obtained by assuming that there is a state $x_{1}$ whose probability is ambiguous, and this ambiguity is compensated by all other states exhibiting constant ambiguity: $d F\left(x_{1}, \theta\right)=p_{1 \theta}$ and there is a cdf $M$ such that for all $x \neq x_{1}$ and for all $\theta=1, \ldots, n, \quad d F(x, \theta)=\left(1-p_{1 \theta}\right) d M(x) . \quad M$ can be interpreted as the conditional loss distribution conditional to $x \neq x_{1}$, which is independent of $\theta$. By an intuitive abuse of language, we hereafter refer to $x_{1}$ as the ambiguous state. In this one-against-all ambiguity context, we know from Proposition 4 that a constant deductible is applied to all losses different from $x_{1}$. We examine the properties of the deductible that should be applied to loss $x_{1}$. 
Proposition 7: Consider the one-against-all ambiguity structure with ambiguous loss $x_{1}$. Suppose that an indemnity is paid in that state. Then the deductible applied to that state is less than the constant deductible applied in all other states. Moreover, the final wealth in that state is smaller than the certainty equivalent final wealth conditional to $x \neq x_{1}$.

Proof: See the Appendix.

The optimal deductible applied to the ambiguous loss is smaller than the deductible applied to all other losses. The intuition is that the ambiguity-averse policyholder is willing to purchase more insurance coverage to compensate for the ambiguity of that loss. This is because final wealth in that state is smaller than its certainty equivalent, so that raising the indemnity in that state has a larger impact on the lower $U_{\theta}$, i.e., those corresponding to the large $p_{1 \theta}$. This insurance strategy reduces the variability of the $U_{\theta}$, i.e., it reduces ambiguity.

applied to the ambiguous loss is 4.37 .

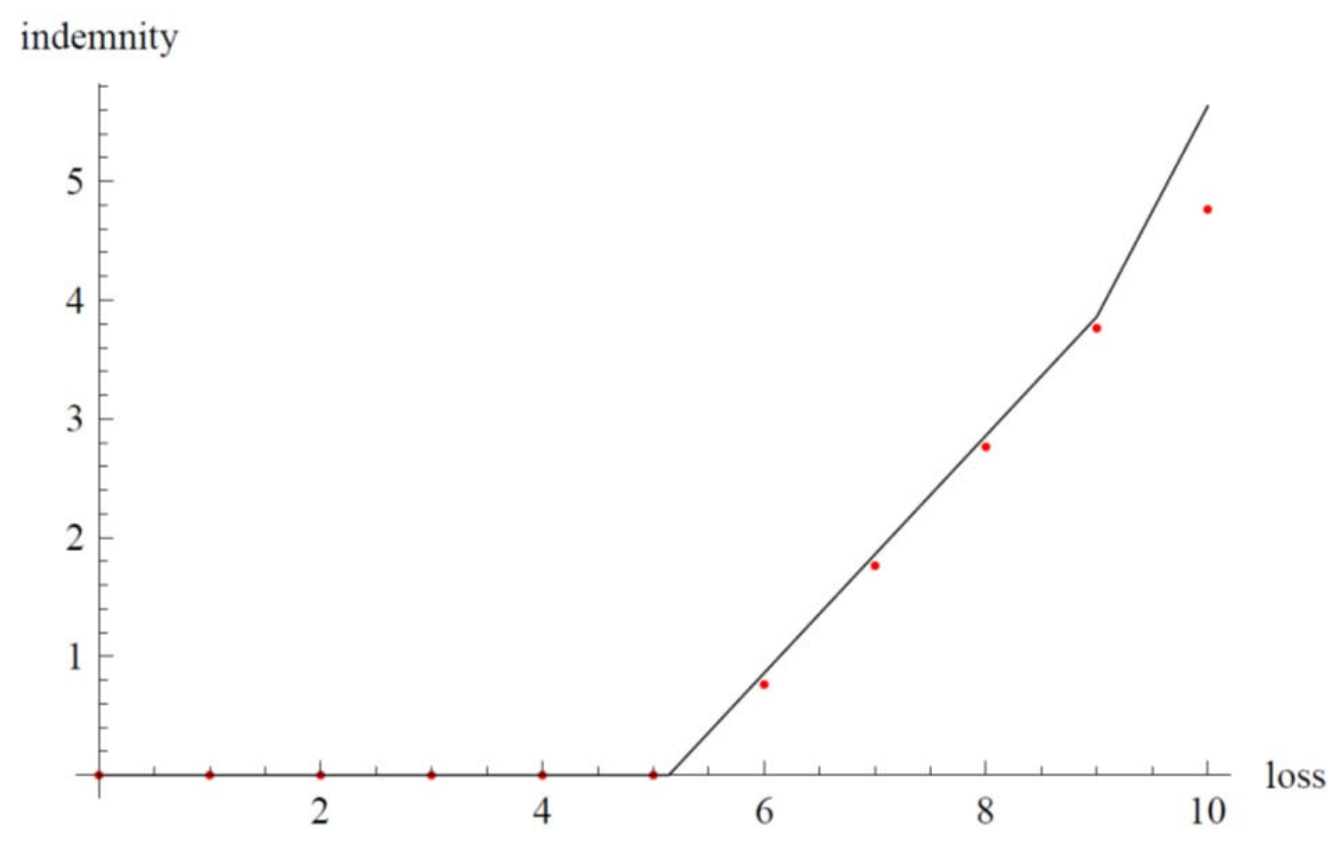

Figure 2: Optimal contract under a one-against-all ambiguity structure. The support of loss is $\{0,1, \ldots, 10\}$. There are two equally likely cdfs, respectively with probabilities $(0.05, \ldots, 0.05,1 / 2)$ and $(0.1, \ldots, 0.1,0)$. We also assume $u(c)=\sqrt{c} / 2, w_{0}=11$ and $\tau=0.11$. The dots correspond to the optimal insurance schedule under ambiguity neutrality, whereas the plain curve is the optimal contract if $\phi(u)=-e^{-u}$.

In Figure 2, we illustrate Proposition 7 with a numerical example. The preferences of the policyholder, the initial wealth and the insurance loading factor are as in the previous 
subsection. We changed the ambiguity structure to fit the one-against-all one. There are two equally likely scenarii with an ambiguous loss $x=10$. In the pessimistic scenario, the probability of $x=10$ is $1 / 2$, whereas the probability of all other losses $x=0,1, \ldots 9$ is $1 / 20$. In the optimistic scenario, the probability of $x=10$ is zero, and the probability of all other losses is $1 / 10$. Under ambiguity neutrality, the optimal deductible is $D^{*}=5.24$. Under ambiguity aversion, the deductible applied to all losses other than $x=10$ is $D^{a}=5.14$, and the deductible

\subsection{Monotone likelihood ratio order}

In this subsection, we examine a "MLR ambiguity structure" in which the conditional cdfs can be ranked according to the Monotone Likelihood Ratio (MLR) order. For the sake of simplicity, we limit the analysis to the case in which there are only two plausible priors, $\theta=1$ or 2. Assuming that the two loss distributions share the same support, we suppose that distribution $F(., 2)$ dominates distribution $F(., 1)$ in the sense that the likelihood ratio $d F(x, 2) / d F(x, 1)$ is non-decreasing in $x$. Notice that because MLR is a special case of firstorder stochastic dominance, this implies that, in the absence of insurance, the policyholder prefers the loss distribution associated to $\theta=1$ than the one associated to $\theta=2$. We show that this ambiguity structure yields an interesting characterization of the optimal insurance contract under ambiguity aversion.

Consider the deductible contract that is optimal under ambiguity neutrality as a benchmark. Because the final wealth is a non-increasing function of the loss under this contract, the MLR nature of the ambiguity structure below $D^{*}$ implies that $U_{1} \geq U_{2}$. By ambiguity aversion, this implies that the policyholder implicitly distorts his beliefs in favour of $\theta=2$. Now, because of the MLR nature of the ambiguity structure above $D^{*}$, this distortion implies that $d G(x) / d H(x)$ will be uniformly decreasing, which is a strong form of pessimism. This effect drives the property that the optimal contract contains a "disappearing deductible", as stated in the following proposition. By "disappearing deductible", we mean that the net loss incurred by the policyholder is decreasing with the loss in the loss domain in which an indemnity is paid. 
Proposition 8: Suppose that the two plausible priors can be ranked according to the MLR order: $d F(x, 2) / d F(x, 1)$ is non-decreasing in $x$. Then the optimal contract is such that no indemnity is paid if the loss is below some threshold $D^{a}$, and the indemnity is positive for all losses above $D^{a}$, with the net loss $x-I(x)$ being nonincreasing in $x$.

Proof: See the Appendix.

To illustrate this result, we numerically solved the optimization problem of the policyholder with the same calibration as in the previous section, except for the distribution of the two equally likely priors. We here assume that the optimistic prior $\theta=1$ has a loss probability of $11 / 66$ for $x=0,10 / 66$ for $x=1$, going down linearly to $1 / 66$ for $x=10$. The pessimistic prior $\theta=2$ is symmetric, with loss probabilities going from $1 / 66$ to $11 / 66$ for losses going from 0 to 10 . Under ambiguity neutrality, the optimal contract has a straight deductible $D^{*}=5.03$. This contract corresponds to the dotted curve in Figure 3. Under ambiguity aversion, a deductible of only 4.04 is applied in case of a loss $x=5(I(5)=0.96)$. For larger losses, the deductible is smaller, going down to 2.78 for the largest loss $x=10(I(10)=7.22)$. The indemnity schedule is almost linear (in fact, it is very slightly convex), with a marginal indemnification rule such that a unit increase in the loss raises the indemnity by $1.25\left(I^{\prime}(x) \simeq 1.25\right)$.

Although the final wealth is increasing in the level of the loss for high losses, the expected utility conditional to the optimistic prior is larger than the expected utility conditional to the pessimistic prior: $U_{1}=5.08>4.78=U_{2}$. By ambiguity aversion, this puts more weight on the pessimistic prior, thereby explaining the increasing willingness to insure large losses. 


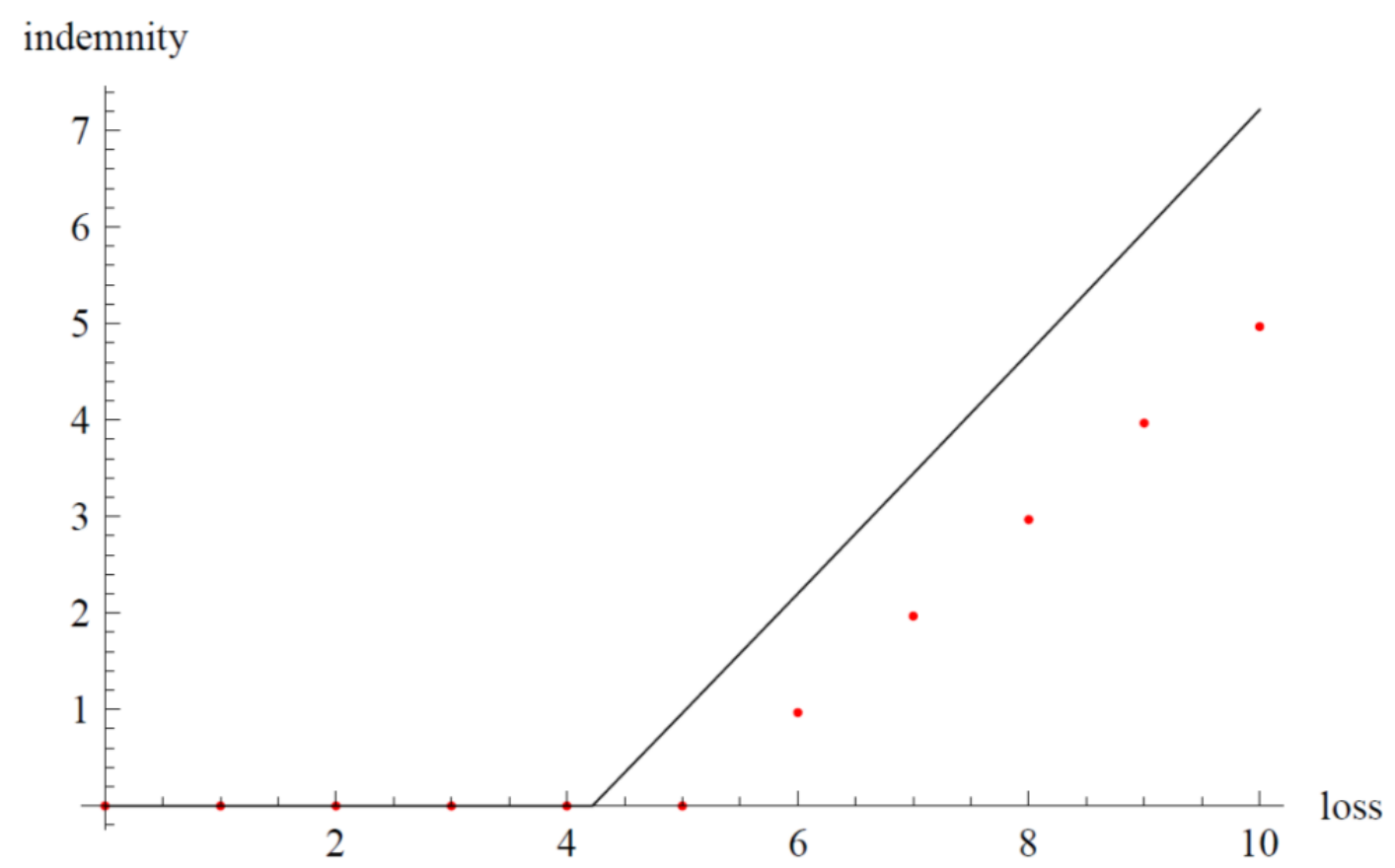

Figure 3: Optimal contract under a two-state ambiguity structure. The support of loss is $\{0,1, \ldots, 10\}$. There are two equally likely cdfs, respectively with probabilities $(11 / 66,10 / 66, \ldots, 1 / 66)$ and $(1 / 66,2 / 66, \ldots, 11 / 66)$. We also assume $u(c)=\sqrt{c} / 2, w_{0}=11$ and $\tau=0.11$. The dots correspond to the optimal insurance schedule under ambiguity neutrality, whereas the plain curve is the optimal contract if $\phi(u)=-e^{-u}$.

\section{Comparative statics of ambiguity aversion}

Up to now, we were mostly interested by the characteristics of the optimal insurance schedule under ambiguity aversion. In this section, we explore the effect of ambiguity aversion on the intensity of the demand for insurance. The intuition suggests that ambiguity aversion should raise the demand to insure ambiguous risks. This intuition is reinforced by the two numerical solutions that we presented earlier in this paper, in which the optimal indemnity is always larger or equal under ambiguity aversion than under ambiguity neutrality, in all states. It is also reinforced by the analysis provided by Alary, Gollier and Treich (2011). As explained earlier, they examined an ambiguity structure in which the probability of loss is ambiguous, but ambiguity is constant in all loss states. They demonstrated that the optimal deductible is decreasing in the degree of ambiguity aversion, as is intuitive. In this section, we show that this is not true in general.

We focus our attention in this section on another case in which the optimal insurance design contains a straight deductible. Namely, we examine the case described in Section 3.2, where the ambiguity is concentrated on losses below $D^{*}$. We know from Proposition 5 that the optimal 
contract has a deductible $D^{a}$ if the degree of ambiguity aversion is small enough. We examine whether $D^{a}$ is smaller or larger than $D^{*}$ in this context. In the following proposition, we show that $D^{a}$ is larger than $D^{*}$ under quite general conditions. ${ }^{2}$ The ambiguity aversion raises the optimal deductible, i.e., it reduces the demand for insurance.

Proposition 9: Suppose that the ambiguity is concentrated on losses below $D^{*}$, i.e., $d F(x, \theta)=d G(x)$ for all $x \geq D^{*}$ and all $\theta=1, \ldots, n$. Suppose also that the degree of ambiguity aversion is small enough so that $I(x)=\max \left(0, x-D^{a}\right)$. The optimal deductible $D^{a}$ is larger than the optimal deductible $D^{*}$ under ambiguity neutrality if one of the following two conditions is satisfied:

1. The conditional distribution functions $F(., \theta), \theta=1, \ldots, n$, can be ranked according to First-degree Stochastic Dominance (FSD);

2. The conditional distribution functions $F(., \theta), \theta=1, \ldots, n$, can be ranked according to the Rothschild and Stiglitz (1970) risk order, and u' is convex.

Proof: See the Appendix.

The intuition of this result is as follows. There is a cost and a benefit to marginally reduce the deductible to get more insurance coverage. Let us first examine these cost and benefit under ambiguity neutrality. The cost corresponds to the increased insurance premium, and the uniform reduction in final wealth. In utility terms, it is measured by the expectation of marginal utility. The benefit of reducing the deductible comes from the reduced risk borne by the policyholder. How does ambiguity aversion modify costs and benefits? Because the ambiguity is concentrated on losses strictly below the straight deductible $D^{*}$, a marginal reduction of this deductible has no effect on the uncovered ambiguity borne by the policyholder. So, it has no effect on the utility benefit of increasing insurance coverage. On the contrary, it raises the utility cost of insurance coverage, which is measured by the expected marginal utility of final wealth using the ambiguitydistorted beliefs:

\footnotetext{
${ }^{2}$ We say that cdf $F(., 2)$ dominates cdf $F(., 1)$ in the sense of FSD if and only if $\int h(x) d F(x, 2)$ is larger than $\int h(x) d F(x, 1)$ for all non-decreasing functions $h$. We say that $\operatorname{cdf} F(., 2)$ is riskier than $\operatorname{cdf} F(., 1)$ in the sense of Rothschild and Stiglitz (1970) if and only if $\int h(x) d F(x, 2)$ is smaller than $\int h(x) d F(x, 1)$ for all concave functions $h$.
} 


$$
\sum_{\theta=1}^{n} q_{\theta} \phi^{\prime}\left(U_{\theta}\right) \int u^{\prime}\left(w_{D^{*}}(x)\right) d F(x, \theta) .
$$

In general, because $u$ is increasing and concave and $u$ ' is decreasing and convex, changes in distribution that raise expected utility also reduce expected marginal utility. Condition 1 or condition 2 guarantees that anti-comonotonicity. It implies that ambiguity-averse policyholders put more weight on scenarii that yield a larger expected marginal utility. They will thus be more reluctant than ambiguity neutral policyholders to sacrifice wealth for insurance.

To sum up our findings in this section, we shown that it is not true in general that ambiguity aversion raises the demand of insurance for ambiguous risks. As claimed for example by Gollier (2011), more ambiguity aversion yields more pessimism rather than more risk aversion. Contrary to the comparative statics of more risk aversion in the insurance problem (Mossin (1968)), the comparative statics of more pessimism is in general ambiguous (Eeckhoudt, Gollier and Schlesinger (1991)).

\section{Conclusion}

We have shown in this paper that smooth ambiguity aversion may have very different impact on the optimal insurance contract depending upon the structure of the ambiguity. Under ambiguity neutrality, the insurance contract with a straight deductible is optimal. If the ambiguity is entirely concentrated on high losses, this contract has the additional advantage to fully eliminate the ambiguity of the distribution of the retained loss. In that case, ambiguity aversion has no effect on the optimal design of the contract and on the global insurance demand. On the contrary, when the ambiguity is concentrated on losses below the deductible, the optimal contract is still with a straight deductible if the degree of ambiguity aversion is small enough, and the demand for insurance is negatively affected by ambiguity aversion. We have also exhibited ambiguity structures that affect the optimal design of the contract. In particular, we have shown that the optimal contract has a disappearing deductible if the multiple priors that characterize the ambiguity can be ranked by the monotone likelihood ratio order.

This paper provides a new illustration of the richness and complexity of decision problems under ambiguity aversion. The effect of ambiguity aversion on decisions is similar to the effect of pessimism. The nature of pessimism entailed by ambiguous probabilities is very sensitive to its structure. Moreover, the ambiguous probability in one state affects the optimal demand for 
insurance in all other states, contrary what we have in the EU framework. This generates new insights to explain actual behaviours. 


\section{Bibliographic references}

Alary, D., C. Gollier, and N. Treich, (2011), The Effect of Ambiguity Aversion on Insurance and Self-protection, unpublished manuscript, Toulouse School of Economics.

Arrow, K.J., (1963), Uncertainty and the welfare economics of medical care, American Economic Review 53, 941-73.

Arrow, K.J., (1965), Yrjo Jahnsson Lecture Notes, Helsinki. Reprinted in Arrow (1971).

Arrow, K. J., (1971), Essays in the Theory of Risk Bearing. Chicago: Markham Publishing.

Arrow, K. J., (1974), Optimal Insurance and Generalized Deductibles, Scandinavian Actuarial Journal 1, 1-42.

Athey, S.C., (2002), Monotone Comparative Statics Under Uncertainty, Quarterly Journal of Economics, 117(1) : 187-223.

Blazenko, G., (1985), The Design of an Optimal Insurance Policy: Note, American Economic Review 75, 253-255.

Carlier, G., R.-A. Dana, and N. Shahidi, (2003), Efficient insurance contracts under epsiloncontaminated utilities, Geneva Risk and Insurance Review 28 (1), 59-71.

Eeckhoudt, L., C. Gollier and H. Schlesinger, (1991), Increase in Risk and Deductible Insurance, Journal of Economic Theory, 55.

Gilboa, I. and D. Schmeidler, (1989), Maximin expected utility with non-unique prior, Journal of Mathematical Economics, 18, 141, 153.

Ghirardato, P., F. Maccheroni, and M. Marinacci, (2004), Differentiating ambiguity and ambiguity attitude, Journal of Economic Theory, 118, 133-173.

Gollier, C., (2001), The economics of risk and time, MIT Press, Cambridge MA.

Gollier, C., (2011), Does ambiguity aversion reinforce risk aversion? Applications to portfolio choice and asset pricing, Review of Economic Studies 78 (4), 1329-1344.

Gollier, C. and H. Schlesinger, (1996), Arrow's Theorem on the Optimality of Deductibles: A Stochastic Dominance Approach, Economic Theory, 7, 359-363. 
Huberman, G., D. Mayers and C.W. Smith, (1983), Optimal Insurance Policy Indemnity Schedules, The Bell Journal of Economics 14, 415-426.

Jewitt, I., (1987), Risk aversion and the choice between risky prospects: the preservation of comparative statics results, Review of Economic Studies, 54, 73-85.

Ju, N. and J. Miao (2012). Ambiguity, learning, and asset returns, Econometrica, forthcoming. Karlin, S., (1968), Total Positivity, Stanford University Press.

Karni, E., (1992), Optimal Insurance: A Nonexpected Utility Analysis, in Dionne, G. (ed), Contributions to Insurance Economics, Boston: Kluwer Academic Press.

Klibanoff, P., M. Marinacci and S. Mukerji, (2005), A smooth model of decision making under ambiguity, Econometrica 73(6), 1849-1892.

Klibanoff, P., M. Marinacci, and S. Mukerji, (2009), Recursive smooth ambiguity preferences. Journal of Economic Theory, 144, 930-976.

Liu, J.-T., M.-W. Tsou, and J. K. Hammitt, (2007), Health information and subjective survival probabilities: Evidence from Taiwan, Journal of Risk Research 10 (2), 149-175.

Machina, M., (1995), Non-Expected Utility and the Robustness of the Classical Insurance Paradigm, in Gollier C. and M. Machina (ed.), Non-expected Utility and Risk Management, Boston: Kluwer Academic Press, reprinted from The Geneva Papers on Risk and Insurance Theory, 20, 9-50.

Mossin, J., (1968), Aspects of Rational Insurance Purchasing, Journal of Political Economy 76, 533-568.

Pratt, J. W., (1964), Risk Aversion in the Small and in the Large, Econometrica 32, 122-136.

Raviv, A., (1979), The Design of an Optimal Insurance Policy, American Economic Review 69, 84-96.

Rothschild, M. and J. Stiglitz, (1970), Increasing risk: I. A definition, Journal of Economic Theory, 2, 225-243.

Slovic P., (2000), The Perception of Risk, Earthscan Publications, London.

Zilcha, I., and S.H. Chew, (1990), Invariance of the Efficient Sets When the Expected Utility Hypothesis is Relaxed, Journal of Economic Behaviour and Organizations, 13, 125-131. 


\section{APPENDIX}

\section{A1: Proof of Proposition 5}

Suppose that

$$
\phi_{t}(u)=u+t \psi(u)
$$

for all $u$ in the domain of $\phi$, with $t \in \mathbb{R}$ and $\psi$ is a smooth increasing and concave function. We examine the properties of the optimal contract around $t=0$, i.e., when the degree of ambiguity aversion is small. When $t=0$, we know from Proposition 1 that the optimal contract has a straight deductible $D^{*}$. It yields

$$
u^{\prime}\left(w_{0}-x+P\right)<\mu(1+\tau)
$$

for all $x<D^{*}$. Under this contract, the presence of ambiguity for losses below $D^{*}$ implies that the conditional expected utilities $U_{\theta}$ vary with $\theta$. Now, consider a positive $t$. The optimal contract solves the set of first-order conditions (10) and (11). By the Implicit Function Theorem, we know that the characteristics of the optimal contract is continuous in $t$ for $t$ close to zero. So are the conditional EU $U_{\theta}(t)$. Define function $K$ such that

$$
K(t, x)=\frac{d G(x)}{d H(x, t)}=\frac{\left(\sum_{\theta=1}^{n} q_{\theta} \phi_{t}^{\prime}\left(U_{\theta}(t)\right)\right)\left(\sum_{\theta=1}^{n} q_{\theta} d F(x, \theta)\right)}{\sum_{\theta=1}^{n} q_{\theta} \phi_{t}^{\prime}\left(U_{\theta}(t)\right) d F(x, \theta)}
$$

for all $t$ and $x$, where $H(x, t)$ is the distorted probability distribution expressed as a function of parameter $t$. Observe that $K(0, x)=1$ for all $x$. By continuity, condition (20) implies that if $t$ is small enough, then it is still true that

$$
u^{\prime}\left(w_{0}-x+P\right)<\mu(1+\tau) K(t, x)
$$

This means that the first-order condition (10) is still satisfied as a strict inequality for all $x<D^{*}$, or that $I(x)=0$ for this subset of losses.

\section{A2: Proof of Proposition 6}


Property $i$ is a direct consequence of Proposition 4, since the degree of ambiguity is constant in all unambiguous states $x \notin\left\{x_{1}, x_{2}\right\}$. Let $D_{0}$ be defined by the following condition:

$$
u^{\prime}\left(w_{0}-D_{0}-P\right)=\mu(1+\tau)
$$

We first show that final wealth must be larger in state $x_{1}$ than in state $x_{2}$. Suppose by contradiction that $w\left(x_{1}\right)<w\left(x_{2}\right)$. This is possible only if $I\left(x_{2}\right)$ be positive. Suppose without loss of generality that $p_{2 \theta}$ is increasing in $\theta$. This implies that

$$
U_{\theta}=u\left(w\left(x_{1}\right)\right)+p_{2 \theta}\left[u\left(w\left(x_{2}\right)\right)-u\left(w\left(x_{1}\right)\right)\right]+\int_{x \notin\left\{x_{1}, x_{2}\right\}}\left[u(w(w))-u\left(w\left(x_{1}\right)\right)\right] d G(x)
$$

is increasing in $\theta$. Because $\phi^{\prime}$ is decreasing, the covariance rule implies that

$$
\sum_{\theta=1}^{n} q_{\theta} \phi^{\prime}\left(U_{\theta}\right) p_{2 \theta} \leq\left(\sum_{\theta=1}^{n} q_{\theta} \phi^{\prime}\left(U_{\theta}\right)\right)\left(\sum_{\theta=1}^{n} q_{\theta} p_{2 \theta}\right) .
$$

Similarly, we have that

$$
\sum_{\theta=1}^{n} q_{\theta} \phi^{\prime}\left(U_{\theta}\right) p_{1 \theta} \geq\left(\sum_{\theta=1}^{n} q_{\theta} \phi^{\prime}\left(U_{\theta}\right)\right)\left(\sum_{\theta=1}^{n} q_{\theta} p_{1 \theta}\right) .
$$

Combining these two equalities with first-order condition (10) - which must be an equality for $x=x_{2}$-- yields

$$
\begin{aligned}
u^{\prime}\left(w\left(x_{2}\right)\right) & =\mu(1+\tau) \frac{\left(\sum_{\theta=1}^{n} q_{\theta} \phi^{\prime}\left(U_{\theta}\right)\right)\left(\sum_{\theta=1}^{n} q_{\theta} p_{2 \theta}\right)}{\left(\sum_{\theta=1}^{n} q_{\theta} \phi^{\prime}\left(U_{\theta}\right) p_{2 \theta}\right)} \\
& \geq \mu(1+\tau) \\
& \geq \mu(1+\tau) \frac{\left(\sum_{\theta=1}^{n} q_{\theta} \phi^{\prime}\left(U_{\theta}\right)\right)\left(\sum_{\theta=1}^{n} q_{\theta} p_{1 \theta}\right)}{\left(\sum_{\theta=1}^{n} q_{\theta} \phi^{\prime}\left(U_{\theta}\right) p_{1 \theta}\right)} \geq u^{\prime}\left(w\left(x_{1}\right)\right) .
\end{aligned}
$$

This implies in turn that $w\left(x_{2}\right) \leq w\left(x_{1}\right)$, a contradiction. Thus, we must have that final wealth in the smaller ambiguous state is larger than in the larger ambiguous state. From (24), this is possible only if $U_{\theta}$ and $p_{2 \theta}$ are anti-comonotone. This implies that 


$$
u^{\prime}\left(w\left(x_{2}\right)\right) \leq \mu(1+\tau) \frac{\left(\sum_{\theta=1}^{n} q_{\theta} \phi^{\prime}\left(U_{\theta}\right)\right)\left(\sum_{\theta=1}^{n} q_{\theta} p_{2 \theta}\right)}{\left(\sum_{\theta=1}^{n} q_{\theta} \phi^{\prime}\left(U_{\theta}\right) p_{2 \theta}\right)} \leq \mu(1+\tau)=u^{\prime}\left(w_{0}-D_{0}-P\right) .
$$

This means that final wealth in the larger ambiguous state cannot be smaller than when applying deductible $D_{0}$ to the corresponding loss, or equivalently, that $D_{2} \leq D_{0}$. At this stage, we know that

$$
w_{0}-D_{0}-P \leq w\left(x_{2}\right) \leq w\left(x_{1}\right)
$$

We now show that $I\left(x_{1}\right)=0$. Suppose by contradiction that $I\left(x_{1}\right)>0$. Because condition (10) must hold as an equality for $x=x_{1}$, and because $U_{\theta}$ and $p_{1 \theta}$ are comonotone, we have that

$$
u^{\prime}\left(w\left(x_{1}\right)\right)=\mu(1+\tau) \frac{\left(\sum_{\theta=1}^{n} q_{\theta} \phi^{\prime}\left(U_{\theta}\right)\right)\left(\sum_{\theta=1}^{n} q_{\theta} p_{1 \theta}\right)}{\left(\sum_{\theta=1}^{n} q_{\theta} \phi^{\prime}\left(U_{\theta}\right) p_{1 \theta}\right)} \geq \mu(1+\tau)=u^{\prime}\left(w_{0}-D_{0}-P\right) .
$$

This would mean that $w_{0}-D_{0}-P \geq w\left(x_{1}\right)$. This inequality is compatible with inequalities in (29) only if $w_{0}-D_{0}-P=w\left(x_{2}\right)=w\left(x_{1}\right)$. The second equality requires that $U_{\theta}$ be independent of $\theta$, in which case a straight deductible is optimal. But the optimal deductible is $D^{*}$ which is by assumption larger than $x_{1}$. This is a contradiction. Thus, $I\left(x_{1}\right)=0$.

Two cases are still possible concerning state $x_{2}$. A first possibility is that $I\left(x_{2}\right)=0$, as illustrated in Proposition 5. We can set $D_{2}=x_{2}$ in that case, thereby yielding $x_{1} \leq D_{2} \leq D_{0}$. Suppose alternatively that $I\left(x_{2}\right)=x_{2}-D_{2} \geq 0$. In that case, condition (29) can be rewritten as $x_{1} \leq D_{2} \leq D_{0}$.

\section{A3: Proof of Proposition 7}

Suppose that $I\left(x_{1}\right)$ is positive, so that condition (10) holds as an equality for $x=x_{1}$. Suppose by contradiction that $w\left(x_{1}\right)$ is larger than the certainty equivalent wealth $w^{m}$ conditional to $x \neq x_{1}$, which is defined as follows: 


$$
u\left(w^{m}\right)=\int_{x \neq x_{1}} u(w(x)) d M(x)
$$

where $w(x)$ is final wealth under the optimal contract. Observe that

$$
U_{\theta}=p_{1 \theta} u\left(w\left(x_{1}\right)\right)+\left(1-p_{1 \theta}\right) u\left(w^{m}\right)
$$

Because $w\left(x_{1}\right)>w^{m}, U_{\theta}$ is increasing in $p_{1 \theta}$. By ambiguity aversion, it implies in turn that $\phi^{\prime}\left(U_{\theta}\right)$ and $p_{1 \theta}$ are anti-comonotone. By the covariance rule, we obtain that

$$
\begin{aligned}
u^{\prime}\left(w\left(x_{1}\right)\right) & =\mu(1+\tau) \frac{d G\left(x_{1}\right)}{d H\left(x_{1}\right)} \\
& =\mu(1+\tau) \frac{\left(\sum_{\theta=1}^{n} q_{\theta} p_{1 \theta}\right)\left(\sum_{\theta=1}^{n} q_{\theta} \phi^{\prime}\left(U_{\theta}\right)\right)}{\left(\sum_{\theta=1}^{n} q_{\theta} \phi^{\prime}\left(U_{\theta}\right) p_{1 \theta}\right)} \\
& >\mu(1+\tau) .
\end{aligned}
$$

Similarly, we know that the optimal deductible $D^{a}$ applied to all losses $x \neq x_{1}$ is such that, for all $x \neq x_{1}$ larger than $D^{a}$

$$
\begin{aligned}
u^{\prime}\left(w_{0}-D^{a}-P\right) & =\mu(1+\tau) \frac{\left(\sum_{\theta=1}^{n} q_{\theta} d F(x, \theta)\right)\left(\sum_{\theta=1}^{n} q_{\theta} \phi^{\prime}\left(U_{\theta}\right)\right)}{\left(\sum_{\theta=1}^{n} q_{\theta} \phi^{\prime}\left(U_{\theta}\right) d F(x, \theta)\right)} \\
& =\mu(1+\tau) \frac{\left(\sum_{\theta=1}^{n} q_{\theta}\left(1-p_{1 \theta}\right)\right)\left(\sum_{\theta=1}^{n} q_{\theta} \phi^{\prime}\left(U_{\theta}\right)\right)}{\left(\sum_{\theta=1}^{n} q_{\theta} \phi^{\prime}\left(U_{\theta}\right)\left(1-p_{1 \theta}\right)\right)} \\
& <\mu(1+\tau) .
\end{aligned}
$$

The last inequality comes from the fact that $\phi^{\prime}\left(U_{\theta}\right)$ and $1-p_{1 \theta}$ are comonotone. Combining inequalities (33) and (34) implies that $u^{\prime}\left(w\left(x_{1}\right)\right)>u^{\prime}\left(w_{0}-D^{a}-P\right)$, or that $w\left(x_{1}\right)<w_{0}-D^{a}-P$. It implies that $w\left(x_{1}\right)<w_{0}-\min \left(x, D^{a}\right)-P=w(x)$ for all $x$. It implies in turn that $w\left(x_{1}\right)$ is smaller than the certainty equivalent $w^{m}$, a contradiction. Thus, $w\left(x_{1}\right)$ must be smaller than $w^{m}$. Under this condition, inequalities (33) and (34) are reversed, which implies that $w\left(x_{1}\right) \geq w_{0}-D^{a}-P$. This means that the deductible applied to $x_{1}$ is smaller than $D^{a}$. 


\section{A4: Proof of Proposition 8}

In the following Lemma, we take the distorted cdf $H$ as exogenous, and we explore the link that exists between the likelihood ratio $d G(x) / d H(x)$ and the design of the optimal contract.

Lemma 1: Suppose that $K(x)=d G(x) / d H(x)$ is non-increasing. Then, there exists a deductible $D^{a}$ such that no indemnity is paid if the loss is below $D^{a}$, and the indemnity is positive for all losses above $D^{a}$, with the net loss $x-I(x)$ being non-increasing in $x$.

Proof: Define function $h$ such that

$$
h(x)=u^{\prime-1}(\mu(1+\tau) K(x))
$$

for all $x$. Observe that this function is non-decreasing in $x$. Define $D^{a}$ as the single root of equation $\quad h\left(D^{a}\right)=w_{0}-D^{a}-P$. For all $\quad x \geq D^{a}$, condition (10) is satisfied $w(x)=w_{0}-x+I(x)-P=h(x)$. Because $h$ is non-decreasing, the net loss $x-I(x)$ is nondecreasing. For all losses $x<D^{a}$, we have $I(x)=0$ and condition (10) is satisfied as an inequality.

In Lemma 2, we link the MLR condition to the property that $d G / d H$ is decreasing when $U_{\theta}$ is decreasing.

Lemma 2: Suppose that $U_{\theta}$ is decreasing in $\theta$ and that $d F(x, \theta+1) / d F(x, \theta)$ is nondecreasing (non-increasing) in $x$ for all $\theta=1, \ldots, n-1$. Then, $d G(x) / d H(x)$ is non-increasing (non-decreasing). This result is reversed if $U_{\theta}$ is increasing in $\theta$.

Proof: We have that

$$
k(x)=\left(\sum_{\theta=1}^{n} q_{\theta} \phi^{\prime}\left(u_{\theta}\right)\right)^{-1} \frac{d G(x)}{d H(x)}=\frac{\sum_{\theta=1}^{n} q_{\theta} d F(x, \theta)}{\sum_{\theta=1}^{n} q_{\theta} \phi^{\prime}\left(U_{\theta}\right) d F(x, \theta)}
$$

is non-increasing if and only if for all $x_{L}$ and $x_{H}>x_{L}$, we have that

$$
\sum_{\theta=1}^{n} q_{\theta}\left(\phi^{\prime}\left(U_{\theta}\right)-k^{-1}\left(x_{L}\right)\right) d F\left(x_{L}, \theta\right)=0 \Rightarrow \sum_{\theta=1}^{n} q_{\theta}\left(\phi^{\prime}\left(U_{\theta}\right)-k^{-1}\left(x_{L}\right)\right) d F\left(x_{H}, \theta\right) \geq 0
$$


We know that function $g(\theta)=\phi^{\prime}\left(U_{\theta}\right)-k^{-1}\left(x_{L}\right)$ is decreasing in $\theta$. Therefore, it satisfies the single-crossing property. Moreover, by assumption, $d F(x, \theta)$ is log-supermodular in $(x, \theta)$. As is well-known (see Karlin (1968), Jewitt (1987), Athey (2002) and Gollier (2001, Proposition 16)), these are necessary and sufficient condition for (36).

Proof of Proposition 7: Suppose that $n=2$ and that $d F(x, 2) / d F(x, 1)$ is non-decreasing (nonincreasing) in $x$. We first show that this must imply that $U_{2}$ is smaller than $U_{1}$. Suppose by contradiction that $U_{2}>U_{1}$. By Lemma 2, it implies that $d G / d H$ is non-decreasing in $x$. It implies that $I^{\prime}(x) \leq 1$ for all $x$ such that $I(x)>0$ since we must have that

$$
u^{\prime}\left(w_{0}-x+I(x)-P\right)=\mu(1+\tau) \frac{d G(x)}{d H(x)}
$$

for these $x$. By risk aversion, we must have that $-x+I(x)$ must be non-increasing. Hence $I^{\prime}(x) \leq 1$. This implies in turn that final wealth $w(x)$ must be non-increasing in the entire loss domain. But because $F(., 1)$ dominates $F(., 2)$ in the sense se of MLR, this implies that $U_{1}$ is larger than $U_{2}$. This is a contradiction. So, it must be that $U_{2}$ is smaller than $U_{1}$. By Lemma 2, this implies that $d G / d H$ is non-increasing. By Lemma 1 , there must exist a deductible $D^{a}$ such that no indemnity is paid if the loss is below $D^{a}$, and the indemnity is positive for all losses above $D^{a}$, with the net loss $x-I(x)$ being non-increasing in $x$.

\section{A5: Proof of Proposition 9}

By Proposition 5, we know that $I(x)=\max \left(0, x-D^{a}\right)$. We need to prove that $D^{a} \geq D^{*}$. The optimal deductible maximizes the following objective function:

$$
W(d)=\sum_{\theta=1}^{n} q_{\theta} \phi\left(\int u\left(w_{0}-\min (x, d)-P(d)\right) d F(x, \theta)\right)
$$

where $P(d)=(1+\tau) \int I(x) d G(x)$ is the premium as a function of the deductible $d$. Under ambiguity neutrality, the first-order condition of this problem can be written as follows: 


$$
-u^{\prime}\left(w_{D^{*}}\left(D^{*}\right)\right)+(1+\tau) \int u^{\prime}\left(w_{D^{*}}(x)\right) d G(x)=0,
$$

with $w_{D}(x)=w_{0}-\min (x, D)-P(D)$. Because the objective function $W$ is concave in $d$, we know that $D^{a} \geq D^{*}$ if and only if

$$
W^{\prime}\left(D^{*}\right)=\sum_{\theta=1}^{n} q_{\theta} \phi^{\prime}\left(U_{\theta}\right)\left(1-F\left(D^{*}, \theta\right)\right)\left[-u^{\prime}\left(w_{D^{*}}\left(D^{*}\right)\right)+(1+\tau) \int u^{\prime}\left(w_{D^{*}}(x)\right) d F(x, \theta)\right] \geq 0 .
$$

Because the ambiguity is concentrated on losses below $D^{*}$, we have that $F\left(D^{*}, \theta\right)=G\left(D^{*}\right)$ for all $\theta=1, \ldots, n$. Thus, inequality (41) can be rewritten as follows:

$$
(1+\tau) \sum_{\theta=1}^{n} q_{\theta} \phi^{\prime}\left(U_{\theta}\right) \int u^{\prime}\left(w_{D^{*}}(x)\right) d F(x, \theta) \geq u^{\prime}\left(w_{D^{*}}\left(D^{*}\right)\right) \sum_{\theta=1}^{n} q_{\theta} \phi^{\prime}\left(U_{\theta}\right) .
$$

Using equation (40), this is equivalent to

$$
\sum_{\theta=1}^{n} q_{\theta} \phi^{\prime}\left(U_{\theta}\right) \int u^{\prime}\left(w_{D^{*}}(x)\right) d F(x, \theta) \geq\left(\sum_{\theta=1}^{n} q_{\theta} \phi^{\prime}\left(U_{\theta}\right)\right)\left(\sum_{\theta=1}^{n} q_{\theta} \int u^{\prime}\left(w_{D^{*}}(x)\right) d F(x, \theta)\right)
$$

We first show that condition 1 in the proposition is sufficient for (43). Under condition 1 , suppose without loss of generality that $F(., \theta+1)$ dominates $F(., \theta)$ in the sense of FSD, for $\theta=1, \ldots, n-1$. Given that $u(w)$ is non-decreasing in $w$ and $w_{D^{*}}(x)=w_{0}-\min \left(x, D^{*}\right)-P\left(D^{*}\right)$ is non-increasing in $x$, we have that $U_{\theta}$ is non-increasing in $\theta$, and $\phi^{\prime}\left(U_{\theta}\right)$ is non-decreasing in $\theta$. Symmetrically, because $u^{\prime}\left(w_{D^{*}}(x)\right)$ is non-decreasing in $x$ under risk aversion, we have that $\int u^{\prime}\left(w_{D^{*}}(x)\right) d F(x, \theta)$ is non-decreasing in $\theta$. Thus, $\phi^{\prime}\left(U_{\theta}\right)$ and $\int u^{\prime}\left(w_{D^{*}}(x)\right) d F(x, \theta)$ are comonotone. By the covariance rule, this implies inequality (43).

We then show that condition 2 is also sufficient for (43). Under condition 2, suppose without loss of generality that $F(., \theta+1)$ is riskier than $F(., \theta)$ in the sense of Rothschild and Stiglitz (1970), for $\theta=1, \ldots, n-1$. Given that $u(w)$ is concave in $w$ and $w_{D^{*}}(x)$ is linear in $x$ in the relevant domain $x<D^{*}$, we have that $U_{\theta}$ is non-increasing in $\theta$, and $\phi^{\prime}\left(U_{\theta}\right)$ is nondecreasing in $\theta$. Symmetrically, because $u^{\prime}\left(w_{D^{*}}(x)\right)$ is convex in $x$ in the relevant domain $x<D^{*}$, we have that $\int u^{\prime}\left(w_{D^{*}}(x)\right) d F(x, \theta)$ is non-decreasing in $\theta$. Thus, $\phi^{\prime}\left(U_{\theta}\right)$ and 
$\int u^{\prime}\left(w_{D^{*}}(x)\right) d F(x, \theta)$ are again comonotone. By the covariance rule, this implies inequality (43). 